14.

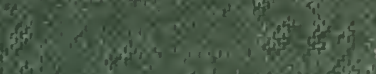

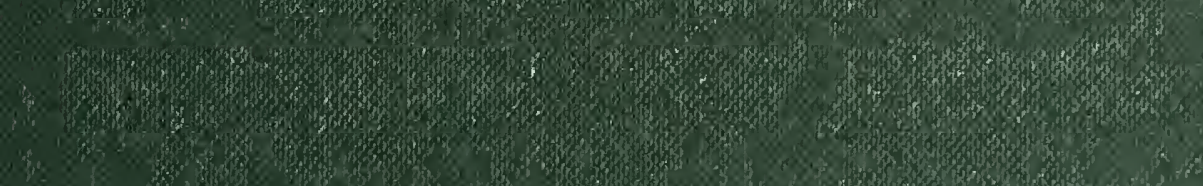

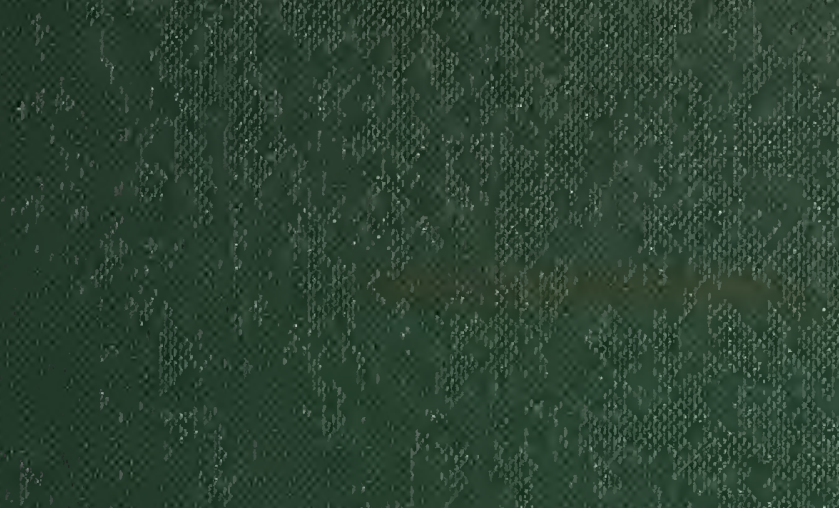

(2)

4.

and

(2)

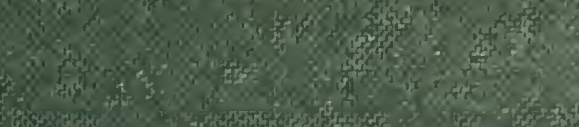

18. 8 


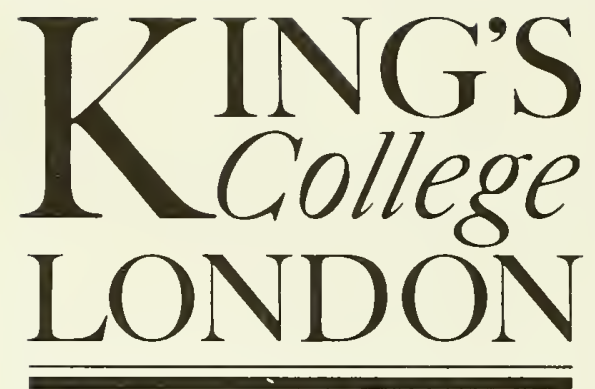

Pukyne Library

Symoke ad ai arrim histerken...

1830

KCSMD QL 953 .PUR

$200824845 \quad 1$

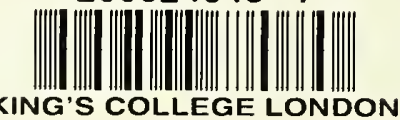


$\sum_{0}^{\infty} \frac{5}{2}$ 



\section{SYM BOLA}

A D

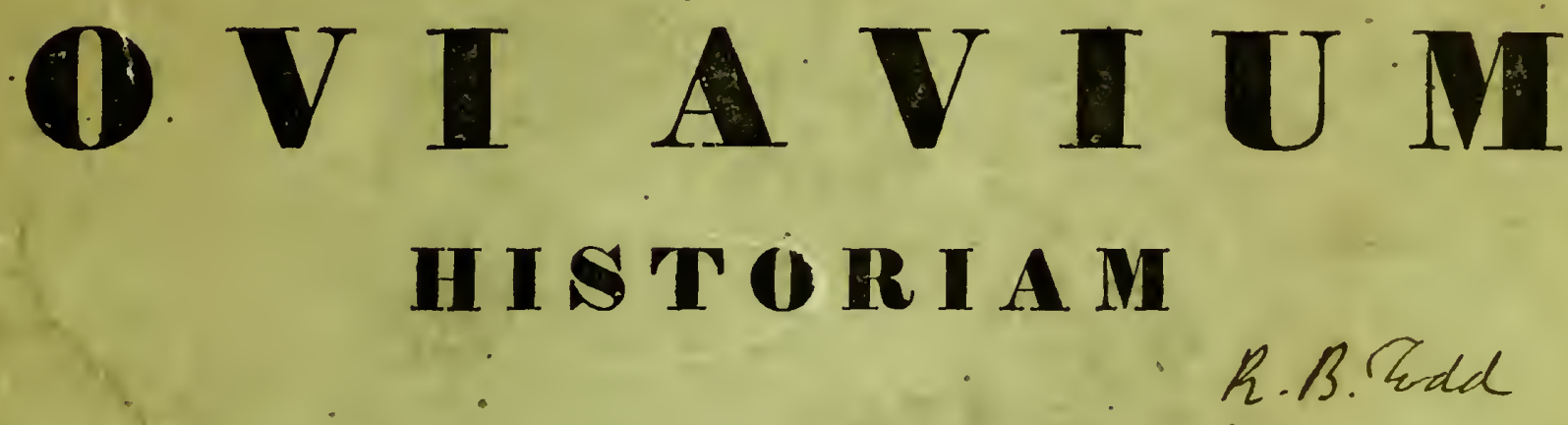

$\begin{array}{lllllllllllllllll}A & N & T & E & I & N & C & U & B & A & T & I & O & N & E & M\end{array}$

A U C T ORE

\section{JOANTF IFANGFITSA PURKINJF}

PIROFESSORR MEDICINAR T. O. VRATISLAVIENST.

ADJECTAE SUNT TABULAE "DUAELITIIOGRAPIIICAE.

TIRSIAE, SUMPTIBUS TROPOHDI TOSSII. MDCCCXXX.

Parisis, apud J. B. Baillièrb. | A matelodami, epud Mülleg et 800.

L ONDINI, apud J. B. BAILliker. VIENNAE, apad J. G. IIRUBNer. 
VERSITY DEPOSITON"

oforgy

R. 112 


\section{Digitized by the Internet Archive in 2015}





\section{SYM BOLA E}

A D

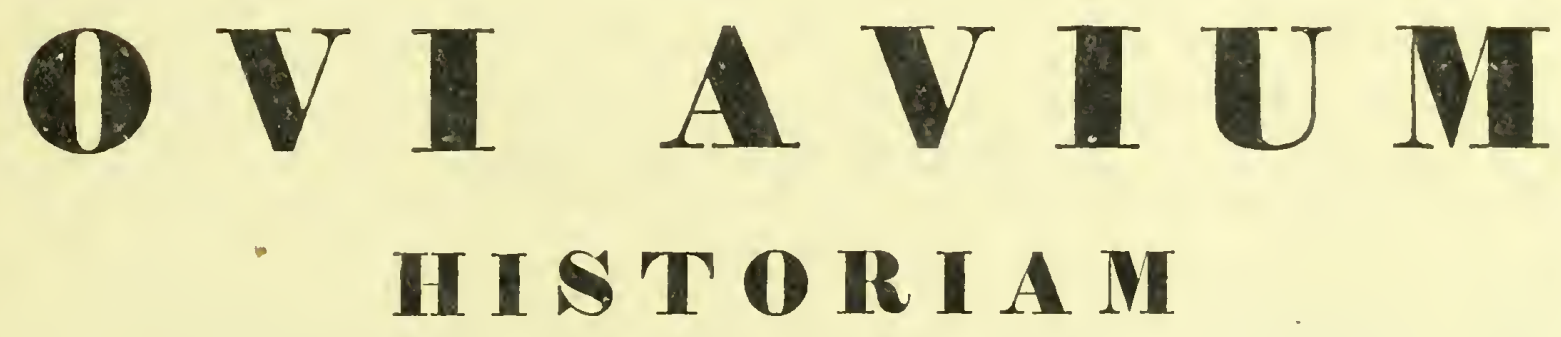

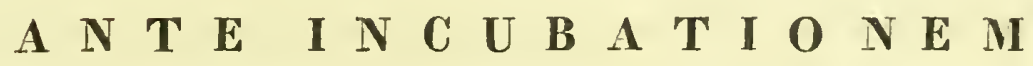

\section{A U C T OR E \\ JOANNE FVANGHEISTA PURKIJI}

PROFESSORE MEDICINAE P. O. TRATISLAYIENSI.

ADJECTAE SUNT TABULAE DUAE LITIIOGRAPHICAE.

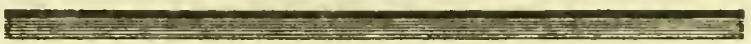

IIPSIAE, SUMPTBUS TEOPOLDI VOSSII.

$\operatorname{MDCCCXXX}$. 
KCSMO QL953.PUR

562821

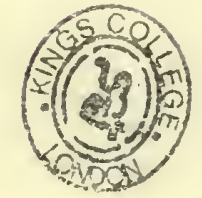




\section{P R A E F T I O.}

Commentatiuncula mea de ovi avium evolutione ante incubationem, quae congratulationibus ab ordine medico Vratislaviensi Blunenвacho oblatis semisaecularia ante quinque annos omine faustissimo celebranti, subjecta erat, typis tunc publici juris non facta est, paucorum tantum manibus revoluta. Nililominus, ob argumenti novitatem atque momentum, a viris compluribus, quos facile proceribus mundi litterarï adnumeres, benignissime excipiebatur, immo etiam fructus peperit, (conf. C. E. a BaEr de ovi mammalium et hominis genesi) qui majoris adhuc momenti videntur, quam argumentum ipsum de quo disserebatur. Nos nunc, a pluribus viris litteratis excitati, laborem hunc recudi fecimus, paucis tantum levibus mutationibus inductis aut notis adjectis, paucioribus omnino, quam desiderandum esset, quum aliis plane objectis hoc tempore animum intenderemus. Exoptandum itaque, ut, dum multiplicatum opusculum plurium naturae curiosorum manibus obversetur, horum forsan quempiam excitet, qui rem 
a nobis inchoatam aut penitius persequatur aut ulterius promoveat. Commendamus praesertim investigationem de subitanea vesiculae germinativae disparitione, dum ovulum ab infundibulo ovarii excipitur. Sed plura, materiem severius indaganti, ultro offerentur. - Liceat denique pagellas hasce iterum vovere Budmenbachio Nestori sane naturae scrutatorum, lustro post semisaecularia faustis auspiciis ad finem vergente.

Dabam Vratislaviae 30mo Maji 1830. 


\section{\$. 1.}

Accuratior inquisitio in cicatriculam ovuli ovarii; detectio ejus vesiculae.

Dum ego prima stamina pulli se evolventis curiosis retro persequebar, tandem, qua via patebat, ad cicatriculam vitelli in ovario retrogressus sum. Ovulum ovarii, uti notum, duplici membrana, quas junctas calycem vocant, instructum est. Harum exterior tenuis et sat firma, sacci aëriferi abdominalis propages, vasa sangnifera ovo adducit et ab eo per petiolum ejus reducit, laxis cellulis interiorem ambiens, donec in stigmate sigmoideo ei penitus accrescat. Membrana interna crassinscula, cujus externa facies asperior ultimas vasorum ramificationes excipit, hujus plaga interna tomentosa, poris sanguiferis aequalibus distantiis notata.

Sequitur dein propria vitelli cuticula, tenuissima, cui stricte vitellus cum cicatricula subest. Totum ovarii ovum, dum ad maturitatem vergit, aliquantulum oblongım est, et ex una parte atque altera paullisper complanatum, quae forma orificio infundibuli oviductus respondet. Minora ovula ad sphaeram accedunt, minima, e tunica ovarii vix media sui parte, exanthematis miliaris instar emergint.

Cicatricula (Hahnentrilt) plagam unam alteramve ovuli notat, petiolo plerumque propior quam stigmati, quamvis etiam sub petiolo ipso aut plane sub stigmate invenerim, nunquam tamen in extremitatibus, ne fors, quum longa axi in initiis oviductus promovetur vitellus, chalaza obtegatur. Internam paginam membranae vielli semper strato tenuissimo et aequabili globulorum obductam inveni, qui sub microscopio magnitudinem et figuram sanguineorum, majorem tamen lis pelluciditatem exlibebant, caeterum organico modo dispositi, non fortuitam indigestamque molem prae se ferentes. In viciniis cicatriculae acervati magis globnli isti zonulam latitudinis undique plusquam semilineae constitumt, cujns internus circuitus cumulum 
eorundem globulorum mammaeformem albescentem, duarum fere linearum, ad interiora vitelli vergentem exhibet $(\boldsymbol{F i g} . \boldsymbol{I}-\boldsymbol{I} \boldsymbol{V}$.) In cacumine cumuli porus ita dictus pellucidus, ab utraque pagina cicatriculae conspiciendus, formae circularis integerrimae offertur, cujus diameter sextam fere p. irtem lineae metitur. Porus hic auctorum in cicatricula ovorum, et eorum, qui in utero atque oviductu latent, nusquam invenitur, neque apud auctores discrimen quoddam inter cicatriculam ovi et ovuli ovarii maturi assignatum reperi. Rem inde curatius inquirere momenti cujusdam esse judicavi.

Sub lente itaque simplici, cujus focus lineam sesquialteram metitur, aciculis chalybeatis cumulum illum mammaeformem centrum versus destruere institui, donec ad porum pellucidum pervenirem. Iterato conatui nil concessum erat, nisi cuticulam quandam tenuissimam dilacerare, quae humorem limpidum includebat. Formam quandam globularem subesse magis auspicari quam clare cognoscere datum fuit. Casus tandem fortuitus multifaria et frustranea juvit conamina.

Dum aquam, cui objecta submersa erant, sugendo aufero, cumulus descriptus, in umbone fundi vasculi vitrei ad siccum redactus, collahitur, et fere diffluit, adeoque porus ejus centralis, uno adhuc fere suo diametro undequaque dilatatur. Haec dum lentis ope perlustro, vesicula formosissima, parte margini pori adlıerens parte libera, haud parum mirabundo mihi offertur. Jam porro eam penitus solvere, et solam atque integram sistere non difficile erat. (Fig. $\boldsymbol{V}-\boldsymbol{I} \boldsymbol{X}$.)

Porum itaque, qui medium cicatriculae cumulum pertundere videtur, vesicula minutissima admodum pellucida occupat, quae substantiae illae globuliferae inmersa, duas tantum facies, unam versus mombranam externam vitelli, alteram ad colliculi cacumen, cratere ibi parvulo circumdata, versus vitelli interiora liberas praesentat, ita tamen, ut in facie externa aliquantulum membranae vitelli allhaereat, ut facile, dum ab ea solvitur, dilaceretur, in interiori vero stratum simplex globulorum ix vicem cohaerentium a colliculo per eam continuetur; atque sic pori falsa species simulatur.

Quodsi hanc vesiculam penitus enucleare successit, reperies eam splıaerae paullulum complanatae figuram referentem, cuticula tenuissima obtectam, lıumorem diffluum limpidissimum continentem. Aliquoties zonu- 
lam ex substantia alba colliculi residuam, in peripheria sua horizontali gerebat, plerumque tamen facili opera penitus liberam ab his sistere contigit, non semel etiam vestigia adlıacrentis membranae vitelli conspicienda erant (Fig. VIII.) Inde in dubio adhuc remanebat, an vesicula colliculi substantiae ad zonam marginalem undique accreta sit, atque ora pori membranis specialibus occludantur. Post permultas vero inquisitiones resiculam integram et singularem esse, dubium non supererat.

Si licet in re tam subtili sensisum manuumque labilitati imaginatione succurrere, existimarem praeterea, ex marginibus pori cumuli membranulam globulosam ad circumferentiam vesiculae accedere, et per utrumque ejus internum et externum hemisphaerium continuari, salva adhuc propria vesiculae cuticula.

Habet itaque cicatricula ovi ovarii partem specialem et sibi propriam, vesiculam sphaericam subcompressam, membranula tenerrima constantem, lympha propria, fors generatrice repletam (iude vesiculam germinativam appellaverim), infossam cumulo albo mammaeformi e globulis composito, in medio pertuso, quem zonula plana albescens circumdat et quae inde in stratum globulorum, faciem internam membranae vitellinae obsidentium, de qua supra sermo fuit, continuatur. Ceterum cicatricula interuae paginae membranae vitelli ita applicata est, ut cum ea semper a vitello secedant.

\section{\$. 2.}

Differentia inter cicu"riculam ovuli ovarii, ovi in oviductu, ovique enixi.

Jam imagiuatio praecox in resicula hac germen foemineum inquilinum, ex quo deinceps pullus evolvatur, auspicabatur. Proximum ilaque erat, cicatriculam ovi exchusi recentis, ante onnem incubationem, summa cura perscrutari, quamnam in eo vesicula nostra metamorphosin subeat.

Novum quidem discrimen se offerebat in eo, quod cicatricula ovi jam exclusi ...embranae vitelli non adhaereat, quum illa ovi ovarii non sine difficultate ab ea discedat, contra vero haec a subjacente vitello facile 
solvatur, illa vero sat arcte vitello accreta sit. Zona porro cicatriculae ovi ovarii tenuis adhuc et arctior in colliculum transit, in quo substantia plastica quasi concentratur; in cicatricula vero ovi exclusi nec non ovi oviductus omnia in latum dispersa sunt, colliculus jam dissolutus, blastoderma ubique spissitudinis aequalis semipellucidum, neque ullum restigium vesiculae offerens. Cicatricula ovi jam efformati duplicem circulum format, quorum exterior vitello adhaeret, interior, qui a priore continuatur, fossula plana (MALPighri fors colliquamento) a vitello separatur. Fossula haec circularis in vitello residet, in medio umbonem albicantem offert (nucleum PANDERI) viscida semipellucida materie obductum, cui granula alba farinae instar inspersa sunt. Eadem granula interiorem etiam faciem adspergunt, excepto centro (Fig. XI.), quod nucleo incumbit quin adhạereat. Blastoderma centrale hic semipellucidum ita, ut interstitium fossulae umbrosum plumbeo colore compareat, nucleus vero albido pelluceat. (Fig. IX - XI.) Vesicula itaque ovuli orarii in ovo enixo dirupta, et in colliquamentum mutata esse videtur.

Sequebatur cicatriculam inquirere, dum aut ovum in utero est, aut vitellus in tractu adhuc oriductus commoratur. In priori eadem reperi quae in enixo. Ast neque in vitello oviductus ullum resiculae restigium aderat, quamris initio quidem, dum adhuc ad infundibulum haeret, colliculi residum aderat, a vitello facilius separandum. Videtur itaque resicula, dum vitellus semilluidus ab infundibulo excipitur, a contractionibus oviductus disrumpi ant dissolvi, atque ejus lympha cum substantia colliculi ita misceri, ut inde collicuamentum illud cum granulis albis enascatm, a residuo colliculi nucleus formetur *.

\section{\$. 3.}

De evolutione vesiculae germinativae (Keimblischen).

Proximum, quod offerebatur, opus erat, eaudem resienlam in cicatriculis minorum adhuc ovulorum inquirere, quod facillimo segotio, prae-

\footnotetext{
Verisimilius jan uunc mili videtur, vesiculan blastodorma centrale mubrosum, de quo prius sermo erat (Fig. XI.), coustituere, ejusque hemisphacria in membranan duplicem dilatari.
} 
sertim in illis successit, ubi substantia vitelli solitam nondum spissitudinem adepta, emulsionis instar diflua, ovulo sub aqua discisso membranam vitelli continno liberam sistebat. Conspicitur tunc in ejus facie interna vesicula prominula diaphana, halone exiii materiei albae globulosae, quae in ovulo maturo cumulum constituit, circumdata. Membrana vitelli consistentiae tunc mollissimae est, et strato globuloso crassiusculo obtecta. Magnitudo resiculae a primis initiis ad matnritatem usque non eadem serie crescit, qua ovula integra. In minimis resicula vix dimidliam partem minor est quam in perfectis, ita ut tunc fere totum loculum vitcllo destinatum adimpleat. Si evolutionis decursum respicias, diceres, resiculam primum esse in ovulo, quod germinando movetur, ast lento gradu, qui a vitello postea ejusque relamentis ad maturitatem usque longe superatur, ita ut, si mathematico modo efferas, duae series aequalis longitudinis formentur ab aequalibus quantitatibus exorsae, quarum una tardius crescit, altera celerius augetur, ut quum membra prima rationem fere aequalitatis servent, ultima pluribus millibus magnitudine ab invicem discrepent. In ovulis jam diametri unius lineae et infra sub lente mediocri macula circularis, umbrosa ob pelluciditatem ab externis conspicitur, resiculae respondens. ( $\boldsymbol{F i g}$. $\boldsymbol{X I I}-\boldsymbol{X} \boldsymbol{V}$.) Facillime deprehenditur in orulis quatuor aut sex linearum, ubi, membranis sub aqua discissis, et extracta vitelli membrana continuo patet. Atque huc referendum puto locum TıEDEMaNNI *, ubi cicatriculam ovulis Graafianis in mammalibus analogam, primum quod in ovulo evolvitur, formamque ejus resiculam compressam aut maculam albam esse dicit. Non equidem adeo difficile negotium est resiculam hanc parvulis in ovis detegere, ulbi substantiae albae globulosie nondum immersa est; in maturis ovis difficilius opera haec succedit. Methodus, quam primo momento casus mihi fortuitus obtulit, me etiam postea maxime juvit. Cumnlo nempe cum poro suo, cautissime a membrana vitelli pistilli ant aciculae incurvae ope sub aqua deterso, ad fundun vasis qui convexus sit oportet, descendere simas; aqua deinde resorpta cumulus dilatabitur, resiculamque revelabit, quod si non primo, saltem iterato ne-

* Zoolngie Vol. III. p. 100. 
gotio succedet. Maxime vero caveas, ne objectum simul cum aqua resorbeas destruasque. Consistentia nempe vesiculae admodum tenera est, ut in minoribus ovulis ad instar bullae aquene a contrectatione levissima dissiliat.

\section{\$. 4.}

Vesicula cicatriculae organon naturale focmineum in avibus.

Jam de significatu vesiculae hujus meditanti, proximus cogitatus se offerebat, esse fors restigium coitus galli, et fore, ut in ovulis gallinarum, quae gallo socio usae non essent, non inveniatur. Ast et in gallinis ejusmodi quae solae degebant, et quarum ova omnia subventanea, documenti caussa, inventa sunt, vesicula non deerat, quamvis, ut mihi videbatur, tenerior, et multo difficilius enucleanda; in aliis vero nullum plane discrimen inveniebatur.

\section{§. 5 .}

De vitelli evolutione ejusque centrali latebra.

De evolutione vitelli pauca tantum adnotanda invenio. Initio quidem vesicula miliaris pellucida e membraura ovarii exanthematis forma numerosissine procrescens cernitur. Vitellus tunc lympha fluida pellucida est a vesicula cicatriculae vix distinguendus. Jam dum ovulum lineae diametrum attigit, turbatur subalbidus vitellus et vesicula per membranas externas transparet. Dum jam pisi magnitudinem adeptum est, vitellus colorem fere lactis spissioris subflavescentis induit. Tunc distinguendi globuli oleosi quamvis rariores, praevalente humore seroso et albuminoso. Ita crescente magnitudine vitellus magis flavescit, donec in maturissimo ovo colorem fere aurantiacum. induat. Vitellus in ovulo maturo, ac dum adhuc in initiis oviductus latet, multo etiam viscidior tenaciorque invenitur quam ante fuit et quam postea evadit dum albumen adducit, et fors partem aliquam ejus fluidam in se trahit. Jam ex eo, quo flavescere incipit, distinguitur in centro ejus substantia albidior fluidiorque, quam in maturis, 


\section{7}

cicatriculae propiorem invenies. Alba haec vitelli substantia in enixis primum ovis luculentissime observatur. Est profecto ea, quae Belliniano quondam problemati * ansam praebuit nec jam solummodo in ovis coctis, sed in recentissimis quoque, et in omni vitello ex eo tempore, quo distinctior apparet, invenienda. Dum nempe forficula Cowperiana, a cicatricula incipiens, partes vitelli sub aqua exscindis, superficies inde ortae zonas tres concentricas diversi coloris praesentant, quarum externa pallidior, media coloris flavi saturioris est, interna rursus pallidior latebram substantiae albae fluidioris circumdans, cujus latex uti in centro residet, rersus cicatriculae nodum canalem subtilem producere videtur*** (Fig. XVIXVIII.) Caeterum centrum hoc album, cum ambientibus concentricis stratis, tantam similitudinem cum cicatriculae forna prae se fert, ut mirum non sit aliquantum praeposterum judicium unum cum altero commiscuisse, praesertim quum in ovo coctione indurato cicatricula, quae albuminosae est substantiae, ab albumine, cui adhaeret, vix distingui possit.

Substantia haec alba in latebra centrali vitelli contenta, sub nicroscopio spectata, constat globulis albis majoribus albumine invicem junctis, ejusdem fere consistentiae et formae, quae est substantiae, colliquamentum cicatriculae occupantis, ita ut compareat, rigere quandam metamorplosis relationem inter locum utrumque; reliquum latebrae lympha satis fluida repletum est. In vitellis coctis commodius est, hanc vitelli compagem inquirere, dummodo persuasum habeas, non coctione primum fuisse productam. Non semel tunc inter primum stratum et medium lamellam sphae-

* Cf. Laur. Belzini opuscula aliquor ad Archibald. Pitchnnium. Lugd. Bat. 1714. p. 14. et porro: Comment. Bononiens. T. II. P. I. p. 85. P. II. p. 369. et sqq. Comın. Pauzi Bapt. Barbi de Belliniano problemate; item Miscell. soc. Taurinens. T. I. p. 3. ct sq. in Comunentario Josnsis FRaxc. Cigna de ovormu elixatormn cicatricula, qui primus Bellinum refutare andet.

* Seriores disçnisitiones dubitationem de hac fabrica milui invexerant. De Baxk quidem in opere suo (Ueber die Entwicklungsgeschichte dev 'Thieve Iter 'Theil Königsb. 1828) nostran de latebra ccutrali vitelli ad theorian de halonibns applicavit, an vero observatione propria confiruarit, unllibi liquet. Nisi jan chalazarmu conformatio ct firma ad vitellum mediante membrana Duтnocherr adplicatio sufficeret ad explicaudum semper situm aecualem cicatriculae superiora versus, laticem ceutralem cum caualiculo suo ad cicatriculan tendeute, perpendiculo analogun dicerem, quo vitel!ns, suapte qunidem intra massau albuminis sursum tendeus, a evolntione ulteriori detineretur, unde cicatricula semper cundem situm supremum servaret. Latex omniuo ille lyıplıaticus vitelli scmioleosa substantia specifice gravior justo assuni potest. 
ricam albuminis coacti inveni, aut a centro albuminosas huc et illuc productiones, praesertim in ovis, quae aliquot horas jam incubationi subjecta erant. In vitello cocto comparet latebram centralem saepe irregularem offerre figuram, et simul cum hac strata concentrica irregularia quoque evadere. In vitello cocto latebra centralis materiam lacteam saporis subsalsi continet, ut inde manifestum sit, chemicam ejus constitutionem a reliquo vitello differre. In pluribus ovis interins vitelli stratum magis oleosum semipellucidum profunde luteum reperi, nec milhi, a quibus conditionibus dependeat, huclum compertum est. Quas ulteriores mutationes subeat vitellus sub incubatione, quomodo lalones circa cicatriculam in eo formentur, quomodo albumen paullatim ei misceatur, seriori disquisitioni relinquenda. Pace lorum, qui microscopicis delectantur observationibus, adlhuc adjicio, vitellum coctum, qui facili opera in pulverem discedit, cumulos elegantissimorım corpuscolorum, crystallorum formam imitantium, exhibere, ita tamen, ut ex diversis vitelli locis deprompti diversam exhibeant speciem.

\section{\$. 6.}

De membrana vitelli.

Vitelli membrana tenerrima aequabilis et pellucidissima est, «neque sub microscopio quantmmcunque dilacerata aut versata ullam organicam structuram prodit. Vesiculam format integerrimam, undique clausam neque ullo poro instructam. In ovulo ovarii initio quidem vix distingues a stratis globulosis, quae a vitello peripheriam versus deponuntur; tunc etiam resicula cicatriculae ita illi juncta est, ut una ab altera sine laesione separari non possit. Etiam in ovo maturiori membrana resiculae cicatriculae huic vitellinae adeo accrevit, ut non raro, dum eam solvendo laceres, restigium circnlare relinquat. (Fig. $\boldsymbol{V I I I}{ }^{*}$.) Atque hita videtur vesicula quasi in membrana vitelli ipsa nidulari et cum ea evolvi.

Quamdiu vitellus in ovario haeret, menıbranae ovili externae relate ad viteilinam admodum teneram adeo crassie sunt, ut opera tantum pertinacissima a membrana vitelii penitus auferri possint quin haec laedatur. 
Operae pretium esset inquirere, quale commercium inter hanc membranam et internam faciem membranae externae vasculosae in ovulo ovarii intercedat, quo fiat ut sanguis secretioni substantiae vitelli inserviat. Hoc certum est, tunc membranae vitellinae paginam internam omnem esse globulis aequalibus pellucidis, ad sanguineorum formam accedentibus obductam, quos facile a globulis oleosis vitelli distinguas.

Membrana vitelli in infundibulo adhuc haerentis rugosa apparet et sat ductilis, ut vitellus, qui tunc viscidior majorisque consistentiae est, figuram valde oblongam assumere valeat, quod necessarium erat, ut vitellus canalem sat strictum infundibuli, quin membrana dilaceretur, ad laxiorem ambitum oviductus permeare possit.

\section{5ith 16.0

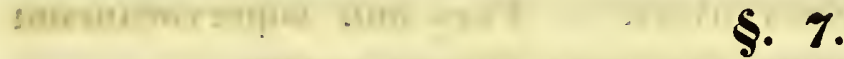 \\ De membranis calicis.}

Jam dum ovulum ovarii ad maturitatem vergit, petiolus ad dimidium fere pollicem producitur, ita ut ovulum maturissimum prae caeteris uropygium versus pertingat, et a subjacentibus intestinis musculorum abdominalium ope ad orificium late patens infundibuli apprimatur. Stigma dein ovuli magis magisque pallescit, et quamvis non extenuetur, ita tamen mollescit, ut facillima demum opera dilaceretur vitellumque effiundat. Membrana tunc externa vasculosa rete laxum e venis tennibus et latis continet, quod utrinque ad stigma surculis in pectines elegantissimos distributis inchoatur. Viletur esse apparatus resorptorius, quo substantia stigmatis sensim sine sensu solvatur vitelloque exitum paret.

Stigma non ultimis primum temporibus in ovulo comparet, sed jam in ovulis uncialibus, quae caeterum semipellucida sunt, lineola albicante turbidiori manifestatur. Interna pagina membranae calicis, quae membranae vitelli contigua est, non, ut quidam volunt et uti adspectus externus docere videtur, velutinae compagis est, sed admodum lacvis atque pellucida, in cujus substantia corpuscula minuta, fors glandulosa, aut rasculorum ampullae aequalibus fere distantiis distribuuntur. 


\section{0}

\section{ร. 8.}

\section{De motibus oviductus et infundibuli et de eorum organo musculari.}

Dum gallinam recens mactatam, quae ovum jam perfectum in utero gerit aperis, intestinaque removes, quo organa ovipara libere pateant, tunc omnem oviductum et uterum motibus continuis peristalticis convulsum deprehendes; praesertim si aquae tepidae submergas, ubi dein fimbriae sinuosae, quae marginem infundibuli coronant, amoenissimis contractionum expansionumque vicibus crispantur. Quodsi, irritando partes, veram oviductus motionum sedem inquiras, facili negotio in mesometrio reperies, quod tunc fibris muscularibus manifestissimis et numerosissimis instructum esse nemo negabit, qui attentus observaverit *. Fors non snpervacaneum erit descriptioni hujus apparatus unam alteramve lineam vovere.

Mesometrium tempore illo quo gallinae ova pariunt nullo modo mesenterii naturam prae se fert, quo intestinis vasa suppeditentur, sed verus musculus est aut potius membrana muscularis, quae omnino vasa etiam ad oviductum distribuit. Distinguitur duplex mesometrium, inferius et superius. Inferius punctum fixum in inferiori facie uteri (si ita vocare licet partem ultimam oviductus ubi testa formatur, et cujus praecipie actione partus ovi perficitur) habet, ubi plexus fibrarum muscularium cruciformis (Fig. XIX.) sat densus ex utroque latere ad uteri ambitum exporrigitur; in parte posteriori uteri quo loco vagina ei inseritur, sacculus musculosus sat laxus formatur, qui sub partu vaginam ab ovo dilatatam ambit et protrusionem ejus adjuvat; antrorsum vero in membranam muscularem fibris reticnlatam flabelli instar dilatatur, cujus peripheria ab insertione oviductus in uterum incipit et insertione posterioris anguli infundibuli in eundem, in se ipsum quasi rediens, terminatur. Alter, anterior nempe infundibuli angulus in ligamentum sat compactum elasticum, fors totum quantum musculare colligitur, quod plerumque ad radicem costae penultimae lateris sinistri insertum est; alias vero minus conspicue circa poros aëri-

- Conf. Ge. Sraxgenere disquisitio circa partes genitales foemineas aviun. Gotting. 1813 c. Tab. pag. 50 . 


\section{1}

feros pulmonum pluribus processibus tenuioribus firmatur. (Fig. $\boldsymbol{X I X}$.) In medio hujus ligamenti pars superior membranae sacci aëriferi abdoninalis circumcirca affixa est. (Fig. XIX.h.)

A ligamento * isthoc lamina mesometrii superioris originem ducit, et ad latus sinistrum columnae vertebralis ex peritonaco simul cum sacco aërifèro exorta, ad uteri parietem dorsalem continuatur. Exinde fibrae musculares ad superiorem partem oviductus descendunt, in cujus superficie attenuantur adeo, ut visu vix colligi queant, et membranam muscularem oviductus tenerrimam formantes, mesometrio inferiori occurruut. In infundibulo membrana interna oviductus glandulosa maxime attenuatur, donec ad limbum ejus penitus dispareat, muscularis vero mesometrii continuatio evolvitur magis, ita ut ille limbus totus quantus e substantia musculari contextus compareat ${ }^{* * *}$. Formant vero tibrae musculares limbi rete tenerrimum maxime complicatum, cujus maculae marginem versus continuo minores crebrioresque evadunt atque in margine subcrenato subtilissimi desinunt. (Fig. $X X$.) Directio priucipalis harum fibrarum transversa ad marginem infundibuli perpendicularis est. In linea media longitudinali infundibuli ita ex utroque limbo fibrae musculares concurrunt, ut ibi suturam transrersim striatam efforment; ubi vero orificium iufundibuli patet, ibi per ejus parietes ad oviductum continuantur. Aliae praeterea fibcillae parallelae marginibus limborum prioribus transrersim intertextae sunt. Fibrillae hae ab extremitatibus infundibuli a ligamento nempe anteriori et ab utero originem ducunt et ommen ejus longitudinem ex utraque parte percurrunt. Hae dum contrahuntur crispationem potissinum limbi efliciust. Atque inde mobilitas nira infundibuli, de qua supra locuti sumus, derivanda. Reliquae fibrae musculares mesometrii transrersim ad oriductum protenduntur, plicis oblongis ac ramificationibus inter se communicant, seque invicem perplectunt, ita tamen, ut interstitia pellucida relinquant, solis membranae serosae laminis occupata, caeterum a nerrorum filis tenuissimis transrersim, a rasis secundariis, quae oviductum adeunt, sub

- Taenia fors Spangenbergir 1. c. pag. 55.

* Contrarium asserere videtur Spangenberg l. c. pag. 55. 
angulo acuto intertexta; vasa vero principalia proxima origini mesometrii cum oviductu parallela decurrunt. Non ubique latitudo mesometrii aequalis est. Prout nempe oviductus tribus flexuris sursum deorsumque sinuatur, mesometrium etiam brevius aut laxius evadit. Fibrillae musculares mesometrii, ubi oviductum jamjam attingunt, ab invicem discedunt, eumque amplectentes membrana musculari tenerrima obducunt; in qua fibras longitudinales, quas auctores adnotant *, frustra quaesiveris, nisi in ultima parte oviductus, ubi uterum adiens per tractum duorum fere pollicum coarctatur **. Hic ab utero fibrae longitudinales distinctissimae continuantur, et in ea regione, ubi oviductus amplificatur, continuo evanescunt. Uterus ipse duplici strato musculorum obtegitur. In externo strato longitudinali directione ab apostomosi oviductus ad vaginae ora excurrunt, in interno membranam, quae testam ovi parat, circulares ambiunt. Etiam in vagina longitudinales fibrae praevalent, circulares potius in ejus sphinctere colliguntur. Vagina et infundibulum, qua canales fere toti musculares, mirum in modum ductiles sunt, ita ut paullatim dilatando, facile plures digitos intrudere possis. Hic ductilitas contractilitati juncta fuit, quum promovendis simul, et ne, oribus proxima, elabantur, retinendis corporibus destinata sint.

\section{§. 9.}

\section{De susceptione ovi ovarii ab oviductus infundibulo ${ }^{*}$.}

Jam ovuli maturi historiam ulterius considerare liceat, si fors datum fuerit lucis quidpiam in rem tam tenebris tectam afferre: Dum vitellus

* Fibrae isthaec longitudinales nil alind sunt, nisi plicae oblongae membranae internae oviductus, albumen secernentis, quae per membranam serosam atque muscularem tenerrimas transparent. Rem ita esse facile comparet, dum detractis membranis illis plicas membranae secretoriae laminas ejus aperiendo spatula complanaveris.

* Isthmum oviductus appellaverim. Haec pars, uti observavi, ad secretionem membranae testae destinata est. Dum ornm albumine jam instructum in isthmi initium intrat, prima parietum resistentia pars intrans compressa finem acutum ovi parat, sequens iter jam dilatatum reperiens in obtusiorem polum rotundatur; in illa arctatione fors etiam conditio mechanica strictioris adcretionis albuminis, quam ligamentum Trederi vocant, quaerenda est.

* Conf. Spangenberg 1. c. p. 59. et sqq. 


\section{3}

justam magnitudinem acquisivit, simul cum eo pedunculus calicis ita crescendo elongatur, ut inter viscera et ovarium retrocedens, infundibuli aperturam attingat. Si ovulum remotis omnibus visceribus ex ovario pendulum consideres, videbis complanatas ejus facies ad dextram sinistramve obversas, stigma vero deorsum spectans. Quodsi jam viscera addas, orulum orificio oviductus ita appriment, ut partes complanatae sursum deorsumque vertantur, stigma vero ab infundibuli orificio exosculetur. Infundibulum ejusque limbum, qui ovulum undequaque lambendo attingit atque amplectitur tunc non esse ignavum, ejus musculosa compages et cognita mobilitas facile evincit.

Rem quidem ita concipio. Os infundibuli musculis suis longitudinalibus dilatatur, ovulumque totum quantum excipit, limbus vero fibrillis, quae ad ejus margines longitudinales decurrunt crispatur et circa ovuli petiolum constringitur. Ita calix ovuli et motibus epistomii et ejus secretis quasi digeritur et quum constrictione et pressione, sanguinis circuitus impeditus, omnem porro nutritionem sistit, extenuatur calix ad disruptionem usque stigmatis, vitellus tunc provolvitur ab infundibulo ulterius excipiendus. Propriae tunc infundibuli fibrillae transversales, quae suturam supra descriptam formant, atque mesometrii superioris et inferioris fibrae initiales, per vices, interiora canalis versus contrahuntur, vitellumque ex aperto calice penitus elidunt, qui ulterius per infundibulum promotus ad oviductum intermedium albumen secernentem perrenit. Infundibulum deinceps rursus aperitur calicemque vacuum expellit. Talis conceptio ovuli ab oviductu processus, quamvis in vivo oculis observare vix unquam contingat, ab anatomica tamen organorum structura sat firmiter comprobari videtur. Turgescentia quadam infundibuli motus hos peragi haud verisimile est, quum neque eam vasculosam spongiosamque structuram prae se ferat, quae organis erectilibus peculiaris est, neque ad eam opus sit recurrere, si musculosum ejus apparatum respicimus.

Situm infundibuli relate ad oviductum in gallinis recens mactatis valde varium inveni. Nunc orificium perpendiculariter dependet, nunc ad sinistrum nunc ad dextrum latus decumbit, neque obversum est oviductui, 
neque necessario ab eo arersum *, sed penes eum protenditur ext:cmitat:bus suis costae penultimae sinistrae uteroque aflixum.

\section{\$. 10.}

Exuberantia sectetionis, organorum generationis marcescentia.

In pluribus gallinis quae ovum perfectum in utero gestabant, ovi maturissimi superficiem jam quasi maceratam reperi, unde inducere ausus sum, limbi actionem quandam digerentem fuisse expertam. Hoc tempore plerumque etiam in abdominis cavo serum flarescens, quasi soluto albumine mixtum deprelıendes, eundemque liquorem non raro inter tunicas calicis effusum invenies, cujus significatum assignare nequeo, fors ab exuberantia nisus sanguinis vitelli secernendi derivandum, quales abscessus lactiformes post partum in feminis. Nonnunquam etiam ovulum maturum ruptum ita inveni, ut vitellus inter membranas calicis effunderetur, splaerica ovuli figura in irregularem commutata.

Quodsi gallinae oviparae non satis nitrimtur, ovorum formatio cessat, et ea ovula, quae ad maturitatem accedebant, singularem mutationem subeunt; rumpitur membrana calicis interna, vitellus in cellulas exterioris membranae effluit, ovulum diminuitur; vitellus albescit et absorbetur; tunc etiam minora ovula sphaericam amittunt speciem et flaccida rugosaque, vitello destituta pendent.

Mirum est quam cito in ejusmodi gallinis, quae ova parere desierant, in mesometriis fibrillae musculares evanescant, ut tunc jam post paucos dies nonnisi vestigia quaedam eorum, lactei coloris, vix conspicua deprehendas. Ast de his multa adhuc accuratius disserenda.

\section{\$. 11.}

De evanescentia vesiculae germinativae.

Vitellus ab oviductu exceptus, chalazis, chalazarum membrana, albumine, membrana testae duplici testaque in progressu suo instruitur.

* Cf. Az. Mosno Versuch einer Abhandlung iiber vergleichende Anatomic a. d. Engl. Göttingen, 1790. p. 80. 


\section{5}

Horum intermam structuram optime perspicies, si evolutionem per tractum oviductus prosequaris.

Primuin quidem, si cicatriculam inquiras, postquam vitellus ab infundibulo jam exceptus est, vesiculam quae prius in cicatricula ovuli descripta fuit, nusquam deprehendes. Pori loco nunc internus circulus blastodermatis visendus est, cumulus in nucleum album mutatus esse videtur, et inter utrunque spatiolum circulare granulis albis conspersum deprehenditur. Si mechanica sufficeret hujus mutationis expositio, dicerem, vitellum ex calice erumpentem et ab infundibulo exceptum, contractionibus hujus talem experiri agitationem, ut vesicula alioqui tenerrima disrumpatur. Ast observationis lacunas opinionum commenta non supplebunt. Recurrendum erit ad observationes iteratas et quidem acutissimas; nisi forte hic natura difficilis ad mysteria non accedenda se subtraxerit.

\section{\$. 12.}

\section{De formatione albuminis chalazarumque.}

Jam me nunc ad ulteriorem ovi formationem converto. In initio oviductus, ubi plicae longitudinales membranae mucosae incipiunt, stratum primum tenue albuminis comparet, vitelli membranam undique obtegens, in oppositis partibus, ubi supra et infra oviductus constringitur, nodulo albuminoso pellucido molli iustructum, a quo utrinque funiculus album inosus, rugis laminae internae oviductus circumdatus continuatur, rudimentum chalazarum. (Fig. $\boldsymbol{X X I}$.) Nullum adhuc tunc vestigium adest tibrarum albarum contortarum, quae centrum chalazarum perfectarum occupant, neque adhuc vitelli membrana a contorsione chalazarum rugis radialibus notatur. Prout motu oviductus peristaltico vitellus ulterius promovetur, stratum super stratum secreti a parietibus albuminis, spirali tractu superadditur. Prima interea albuminis ad vitellum lamina spissescit, et in membranam sat solidam, membranae vitelli arctissime adhaerentem commutatur, a qua interna albicantia stamina chalazarum continuantur. Vitellus adeo cum circumdaute albumine circa axem suum mobilis, chalazás, membranae suae affixas, torquendo ita format, uti filum de colo carpitur, 
et in se ipso pluries atque iterum contorquetur. Hinc in extrema superficie albuminis ulterius efformati laminas in gyros circumactas reperies. Haec non imaginationis figmenta esse optime liquebit, quodsi ex ultima fere oviductus parte, ovum albumine jam perfecte obductum eximas, et aquae fontanae frigidae recentissimae immersum, post horulam inquiras. Albumen, quod primum pellucidum hyalinum erat, ab aqua turbatur paullulum et albescit, et strias in superficie sua offert, quae ex fine obtuso a dextris sinistram versus procedentes, spirali modo ad finem acutum excurrunt. Has, si volsella arripias, lamellatim eadem directione discedunt, donec omne albumen ad chalazas usque evolvatur. (Fig. XXII.) Jam dum fere ad chalazarum membranam, vitellum obtegentem perveneris, ultima strata mollissima sunt, ut fere vacuum spatium credas; residuum tunc albuminis, quod ad chalazas suapte discedit, mitrellarum instar ex chalazis pendulum. Ex his colligere licet, Fabricium ab AQua pendente rectam omnino ideam de formatione albuminis et chalazarum proposuisse, neque adeo haec ex vitelli membrana germinare et plantarum instar vegetare quis crediderit.

\section{\$. 13.}

\section{De membrana chalazifera DUTROCHETI.}

Membrana chalazifera DutrochetI *, initio quidem albuminosa, in progressu per oviductum solidescit, et denique eandem fere naturam induit, quae est membranae vitelli. Internum albicans chalazarum filum hujus continuatio est, atque a membrana isthac ad interna chalazarum quasi canalis in se plicatus torsioneque clausus protenditur; ac si saccum membranaceum utrinque apertum sphaerae induas et in utroque polo torquendo occludas.

Membrana vitelli propria salva atque integra manet, uti in ovarii ovulo exstitit, neque per eam communio datur cum canali chalazarum, quo albumen ad interna vitelli perducatur. Canalem, quem LEVEILLE **

- Journal de Physique T. 88. p. 170.

* Reils Archir B. IV. p. 418. 
vidit, facile demonstrabis, quodsi sub aqua chalazam transversim perscindas. Sed non in quavis succedet, in ea tantum, ubi bis in se torta est, quo torsiones ad se invicem applicatae canalem internum, quasi spinam cochleae, necessario formant, qui tamen nullo modo cum vitello communicat. (Fig. $X X I I{ }^{*}$.) Videtur membrana vitelli, quamvis sub microscopio porosa non appareat, humoribus nutritiis undique permeabilis, uti fere in ovulo ovarii accidit, ubi a membrana interna vasculosa sanguinem excipiens, hunc ad interiora ducit, et in vitelli substantiam commutat; idem accidere videmus incipiente pulli evolutione, ubi albumen supra cicatriculam, crateris forma evanidum, ad interna discedit. Simile itaque haud sine dubio per omnem hujus membranae superficiem albumini contingere, facile conjicimus, quin opus sit suctorios canales tingere. Non difficile est, membranam chalaziferam a subjacente vitellina, loco quo chalazae inseruntur, separare, ubi etiam pars ejus, in funiculum torta, in membranam explicatur. Ad hoc feliciter efficiendum, apprehendas volsella chalazam prope suam ad vitellum radicem, et ex vitello sub ea pendulo simul partem membranae vitellinae exsciudas eluasque, dein sub aqua, leute mediocri munitus, membranas ambas (vitellinam et chalaziferam) volsellis duabus, se invicem juvantibus, divellas. Dantur ova, in quibus libero oculo haec patent, membrana chalazifera, laxe tantum ad insertionem chalazarum vitellinae applicata. (Fig. XXIII.) Dantur alia, ubi funiculus non ad vitelli membranan, sed ad interpositum globum substautiae albuminosae, membrana chalazifera obductae, terminatur.

$$
\begin{gathered}
\text { \$. } 14 . \\
\text { De zona albicante in vitelli superficie. }
\end{gathered}
$$

In plurimis ovis, ex uno alterove funiculo per membranam chalaziferam, in superficie vitelli, una (Fig. $\boldsymbol{X} \boldsymbol{X I V}$.) aut plures striae albidae, nunc ex una nunc ex altera parte aut per omnem vitelli ambitum, modo inconstanti, contiuuautur, quasi ibi menbrana incrassata aut in se plicata esset. Sunt istlaec, quae VICQ d'AZYR * zollam albicautem rocat, et quae

- Oeurres T.IV. p. 392.

Purkinje Symbolue. 
in diversis ovis, diversissime disposita, nec, ut quidam adducunt, constantem formam et ad vitellum rationem prae se ferendo, nil nisi vestigium manifestius membranae chalaziferae praesentant. Plerumque striae hae a minori chalaza in parte obtusiori excurrunt, quae nonnunquam oblique torta in eas transit. Saepius plures sunt numero, partem aut omnem vitelli circumferentiam ambientes plerumque cicatriculam evitant, nonnunquam tamen per mediam procedunt. Si membranam chalaziferam detrahas, plicas earum non raro detergere succedit; plerumque tamen non plicae sunt, sed incrassata, tendinum instar argenteum reddens splendorem, substantia membranae chalaziferae.

Nonnunquam in parte obtusiori ovi solae ejusmodi striae reperiuntur, neque ullum vestigium chalazae *; tunc etiam albumen in obtuso fine ovi valde diminutum cernitur et fere omne in fine acutiori accumulatur. Hujus si genesin in oviductu inquiras, diceres, a secretione albuminis nimium praecipitata derivandam esse, quo fit, ut pars vitelli in via per oviductum anterior, membranam ejus ad secretionem copiosam irritet, quae vero cito exhauritur, ut dum in eundem locum finis vitelli subsequens promovetur, parum adhuc, quod secernatur, supersit. Haec in casu insolito accidentia, rationem etiam reddunt, cur in statu normali, in parte ovi obtusiore minor adsit chalaza, minor etiam copia albuminis.

\section{\$. 15.}

De albumine ita dicto tertio deque zona chalazarum.

De albumine tertio inquirens, caveas ne in eandem cum aliis ** illusionem incidas, quae et mihi longins imponebat. Verum quidem est, albumen circa funiculum interiorem albicantem chalazae spissius esse, et fere ad gelatinam accedere, quod si, ut melius distinguas, tertium appellare velis, non obsum, quamvis paullatim, ad extremitates chalazarum, in albumen reliquum ita transeat, ut fines assignari nullo modo possint; ast

- Conf. Léveillét l. c. p. 416.

or Comes ab Thedern diss. sistens ovi aviun historiae prodr. Fig. II. et. III. 


\section{9}

quem auctor citatus cumulum circa chalazas depingit, continuatum in zonae formam ad extremitatem ovi alteram, atque et hic chalazam ambientem, et quod albumen tertium designat, revera non est, sed circumferentia albuminis secundi, oblique visi, vitello chalazisque strictius appressi, reliquo albumine in fundo vasis proprio pondere subsidente et effuso; quod, mea quidem persuasione ob illusionem opticam, circumferentiam peculiarem praesentare videtur. Similem illusionem videntur passi, qui de normali zona, chalazarum cicatriculam ambiente, et sphaerulam vitelli ratione 80: 100 amplectente, loquebantur.

\section{\$. 16.}

Membrana propria albuminis negatur.

Nominare quoque solent auctores membranam albuminis propriam; ast, an eam quis viderit, valde dubito, nisi prius arte paraverit, quum aqua recens coagulando in albumen agat ubicunque tetigerit. Optime quidem ejusmodi membranam manifestam reddere sequenti modo mili successit. Orum recens testa exclusum sub aqua frigida aliquot horas mersum facias. Albumen tunc superficiem solidiorem albescentem acquirit. Auferas nunc sugendo aquam albumen ambientem, tubulumque exilem in albuminis substantiam ad chalazae usque radicem intrudas et leniter inspirando albumen infles; intumescit paullatim in bullam, et sexies fere volumine ovi augetur, ejusdemque formam, quodsi bene opera successit, serrat integerrimam. Tunc iterum aquam leniter affundas, ut ovum, libere natans, inferiora quoque versus, ubi fuudus vasis compresserat, expandatur. Vides tunc vitellum cum chalazis liberum fere, nisi lae extremitatibus suis in albumen utrinque transirent, sacco magno inflato oviformi sat solido in superficie aquae fluctuinte inclusum. Si tunc superficiem leniter incidas, membranam crassiusculam albicantem ab ea detrahere licet, cujusmodi nova iterum formatur quoties aquam, aut spiritum vini aflundas. 


\section{\$. 17. \\ De albumine fluido.}

In ovo, dum adhuc in oviductu versatur; albumen primum seu fluidum non comparet. At neque in ovo uteri, quamvis jam testa obducto, distinguitur. Albumen tunc secundum membranae testae adhaeret neque ligamentum albuminis in parte acutiori discernitur. In ovo mox excluso albumen hoc, dum ovum sub aqua aperitur, non diffluit, sed ovi forma, paullulum pellucidius, albumen secundum circumdat, quamvis jam tunc multo fluidius. Videtur in formatione hujus albuminis aëris atmosphaerici actio, uti dum crassamentum sanguinis formatur, plurimi esse momenti; fors etiam pressio aëris fluidiorem partem ex interstitiis lamellarum albuminis secundi peripheriam versus elicit. Certum est, albumen secundum, dum fluidius illud ex eo nondum separatum est, multo facilius ab aqua recenti, cui immittitur, penetrari et lamellosam suam structuram prodere. Albumen hoc fluidum eximia plasticitate donatum est, uti comparet, dum ovum sub aqua recenti aperias, ubi diffluxum brevi in membranas fibrasque tenerrimas condensatur.

Quodsi ludum amoenum tibi parare velis, aperias in patella ovum quin aquam adhibeas; tunc facile distinguetur albumen fluidum, quod in fundo vasis diffusum est, a spissiore, quod vitellum circumdat; partem hujus deinde tubulo exili insugas et aquae recenti, aut gallarum solutioni aqua dilutae instilles, aut tractim subsidendove effundas: formantur tunc elegantissimi sacculi, vasa, membranae, cellulae, fibraeque, ut vix a veris organicis productis distinguere valeas, proditurque adeo processus, quo natura plasmata sua struere videtur.

\section{\$. 18.}

De chalazarum genesi atque usu mechanico.

Vitellus dum albumine in oviductu induitur, paullatim ulterius promovetur, donec ad isthmum perveniat. In progressu suo non ecquidem eodem modo movetur, quo intestinorum musculosis parietibus contenta pro- 


\section{1}

pelluntur. Motum lunc potissimum fibrae musculares mesometriorum perficiunt, dum oviductum huc et illuc traliendo sinuant torquent atque constringunt. Pars tunc menbranae ejus mucosae, quae vitellum ambit, albumen membranarum forma secernit, quod continuo luic adglutinatur, pars vero ante et post vitellum in se ipsa contracta albumini secreto funiculi tantum formam concedit, qui spiraliter promotus in se ipso contorquetur et in utroque ovi polo accumulatur; plerumque hac torsione ad radicem qua vitello applicatur redit et ibi in reliquo albumine disparet. (Fig. $X X I V$.) Quod a latere axis vitelli chalazae inserantur, servitium in eo praestare videtur, quod punctum gravitatis vitelli semper in suam partem trahant, quo fit, ut cicatricula semper supremum vitelli locum occupet, proximum calori incubantis gallinae exponendum.

Dum ovum apparatu mechanico celerrime circumagis, chalazae inde numerosiores contorsiones non acquirunt, ast ligamentum albuminis detortum a testa abripitur.

\section{\$. 19.}

\section{De formatione membranae testae.}

Membrana testae ad albuminis superficiem externam in ultimo primum tractu oviductus, qui inter ejus isthmum et uterum versatur, accedit. Prima intrat pars ovi acutior, membranamque induit, ita ut in obtusiori albumen saepe. nudum adhuc visendum sit. Sub hoc nisu tractus isthmicus adeo expanditur, ut plicae membranae interioris penitus deleantur atque maxima tensio habeatur strictioris plane partis oviductus, ubi fors major irritatio membranae succum densiorem quo membrana paretur, elicit. Notanda porro interruptio brevis plicarum muciferarum plane sub isthmi strictura, quo intervallum inter secretionem albuminosam et membranaceam efficitur et fors conditio sistitur formandi ligamenti albuminosi in parte ovi acutiori. Membrana testae duplex paratur, interior fibris microscopicis rectis contexta est, exterior texturam peculiarem non offert *. (Fig. $X X V$.)

* In ovo Colubri Natricis stratum hoc interi's fibris undulatis formosissimis constat. conf. G. R. $u$. L. Ch. Treviranus verm. Schrifien Bd. 1. pag. 142.

Purkinje Symbolice. 


\section{2 \\ \$. 20. \\ De situ ovi in utero atque de testae formatione.}

Situm ovi, dum adhuc in utero recens est, semper talem inveni, ut pars acutior vaginam, obtusior basin spectaret, in ovo vero penitus formato, ubi jam nisum ad partum expertum est, nunc obtuso nunc acuto fine vaginae oribus appositum reperi. Fors tunc sub nisu ad partum ovum saepius volvitur donec situm commodum acquirat.

Dum testa formatur, reperis membranam ejus primum minutissimis micis calcareis, fere aequalibus, polygonis aspersam, (Fig. XXVI. XXVII.) quae dein cumulantur et concrescunt, interstitiis inconspicuis relictis, quae transpirationi inserviant.

Quodsi adeo oviductum ab ejus infundibulo usque ad orificium ejus in cloacam apertum consideres, diversas ejus portiones sequentibus functionibus destinatas reperies: Infundibuli orificium e calice exclusum vitellum excipit; sequens dein tractus oviductus, qui fere quartam partem longitudinis ejus efficit, parciorique apparatu glandulari instruitur, membranam Dutrochetr intimaque stamina chalazarum secernit; ulterius canalis membrana interna parenchymate muciparo satis incrassato per duas fere quartas partes totius et ultro obducitur, albuminisque largum indumentum vitello suppeditat; isthmus deinde oviductum coarctat, unde in tractu subsequenti, tertia fere parte totius, ad uterum usque membranae testae albumen obducunt; uterus dein testae secernendae inservit; vagina per orificium externum et cloacam ovum excludit. Hos organorum fines ad oculos spectare licet, neque opus hypothesium adminiculo ad imaginem naturae penitus exstruendam. 


\section{EXPOSITIO TABULARUM.}

Tab. I.

Fig. I. Particula membranae vitelli cum cicatricula ei adhaerente, in cujus centro porus pellucidus conspicitur.

Fig. II. Idem objectum lente vitrea adauctum; in poro vesicula conspicua.

Fig. III. Cumulus cicatriculae qui ad interiora vitelli cernit; in ejus cacumine crater exiguus, interna pori apostomosis.

Fig. IV. Idem cicatriculae cumulus ex opposito visus.

Fig. $V$. Cumulus in fundo vasis aqua resorpta diffluus, porus inde dilatatus vesiculam in medio manifestat.

Fig. VI. Vesicula distinctius visui sistitur, substantia colliculi dimidia parte ablata.

Figr. VII. Vesicula, inflexa cicatricula, in epigrapho visa.

Fig. VIII. Vesicula parti membranae vitellinae adhaerens.

Fig. $V I I I^{*}$. Pars membranae vitelli cum vestigiis zonulae et vesiculae dilaceratae.

Fig. IX, Vesicula cum colliculo in medio discissi.

Fig. $X$. Cicatricula aucta ovi jam enixi, cum duplici circulo blastodermatis, et nucleo in centro albicante.

Fig. XI. Eadem cicatricula sublato interno circulo blastodermatis, quo cavum colliquamenti cum nucleo nudo et granulis farinosis conspiciatur.

Fig. XII. Ovulum ovarii non naturum, in quo cicatriculac vestigium cernitur.

Fig. XIII. Eadem cicatricula ex ovulo extracta parti membranae vitellinae adhaerens.

Fig. $X I V$. Ovulum adhuc minus ovarii cum nota vesiculae.

$\boldsymbol{F i g} . \boldsymbol{X} \boldsymbol{V}$. Idem discissum et auctum, vesiculam continens.

Fig. $X \boldsymbol{V I}$. Vitellus a cicatricula incipiendo perpendiculari directione divisus. In centro latebra liquorem album continens, ex qua canalis ad cicatriculam continuatur.

Fig. XVII. Vitellus horizontali directione discissus, ubi strata diversicoloria substantiae vitellinae cernuntur. 


\section{4}

Fig. XVIII. Pars vitelli cum canali ad cicatriculam.

Fig. XXI. Vitellus cum primo strato albuminoso unde membrana chalazifera exoritur, adnexis primis initiis chalazirum quales supra et infra inter plicas oviductus reperiuntur.

Fig. XXII. Repraesentat albuminis vitellum ambientis structuram lameliosam.

Fig. XXII* Pars chalazae abscissa, in cujus segmento canalis conspicitur ex interni chalazae fili spiris exortus.

Fig. XXIII. Pars membrauae vitellinae cum adnexa membrana chalazifera in chalazam continuata.

Fig. XXIV. Vitellus cum suis chalazis et zonula albicante Vıca d'Azyrı.

Tab. II.

Fig. XIX. Repraesentat oviductum gallinae cum infundibulo utero et parte vaginae: $a$. Uterus ovo gravidus. b. Vagina. c. Fibrae musculares quae ad vagiuam, ad latera uteri et ad mesometrium inferius vergunt. $d d d$. Mesometrium inferius; discernuntur in eo fibrae musculares et vasa directione parallela cum oviductu decurrentia. $e$. Limbus fimbriatus infundibuli. $f$. Infundibuli os. $g$. Ligamentum infundibuli radici costae penultimae lateris sinistri affixum. . Pars abscissa sacci aëriferi ligamento inserti.

Fig. $X I X^{*}$. Pars oviductus posterior ubi in isthmum transit, cum adnexo mesometrio fibris muscularibus nervis atque vasis pertexto.

Fig. $X X$. Pars fimbriae infundibuli aucta reticulis muscularibus pertexta.

Fig. $X X V$. Pars membranae testae cujus margo structuram tomentosam offert.

Fig. $X X V I-X X V I I$. Particulae testae cum subjacente membrana dum crystallisando formatur.

Fig. $\boldsymbol{X} X \boldsymbol{V I I I}$. Oviductus formam dilucidat. 



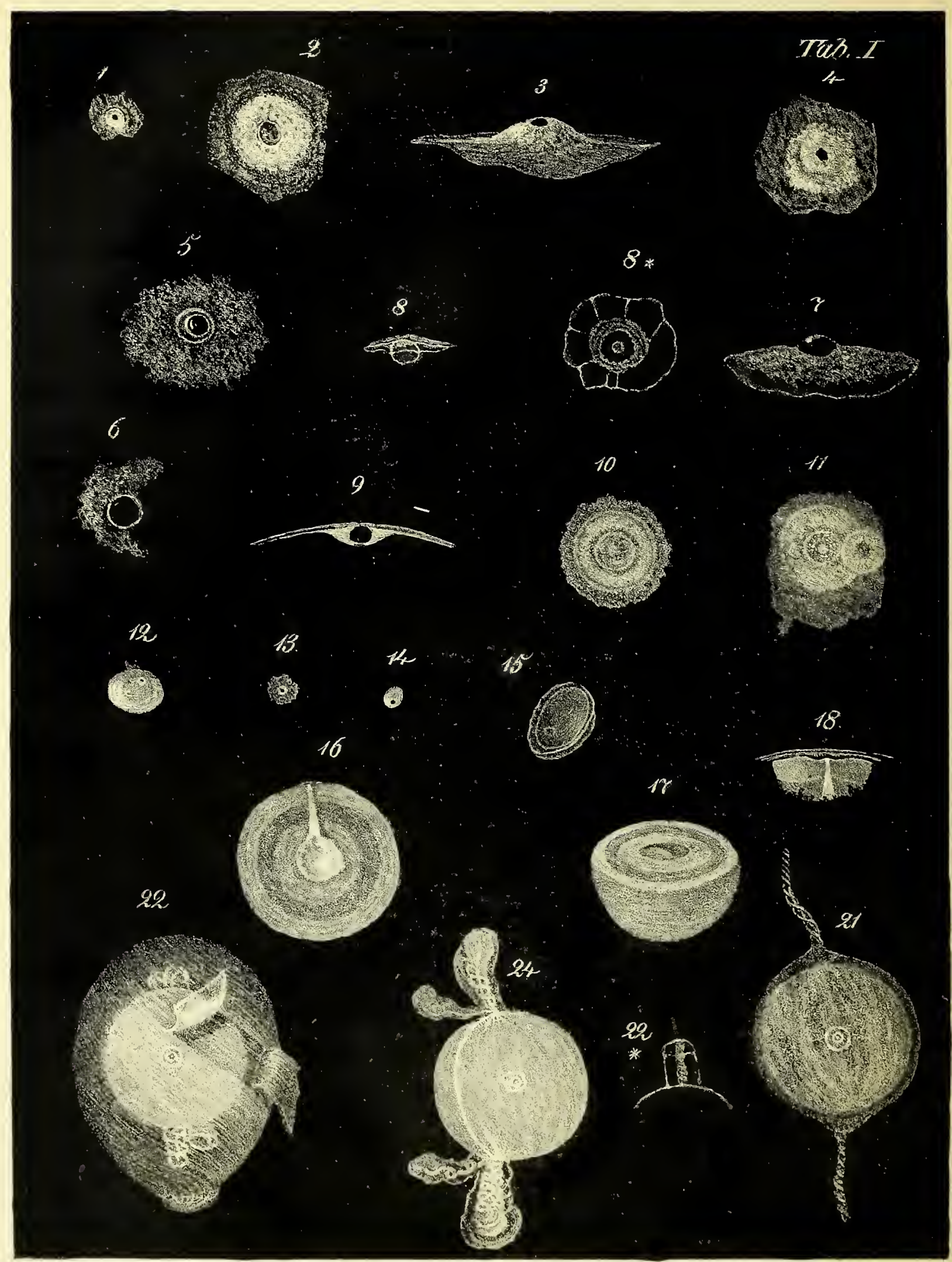

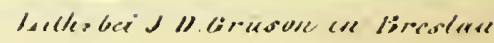




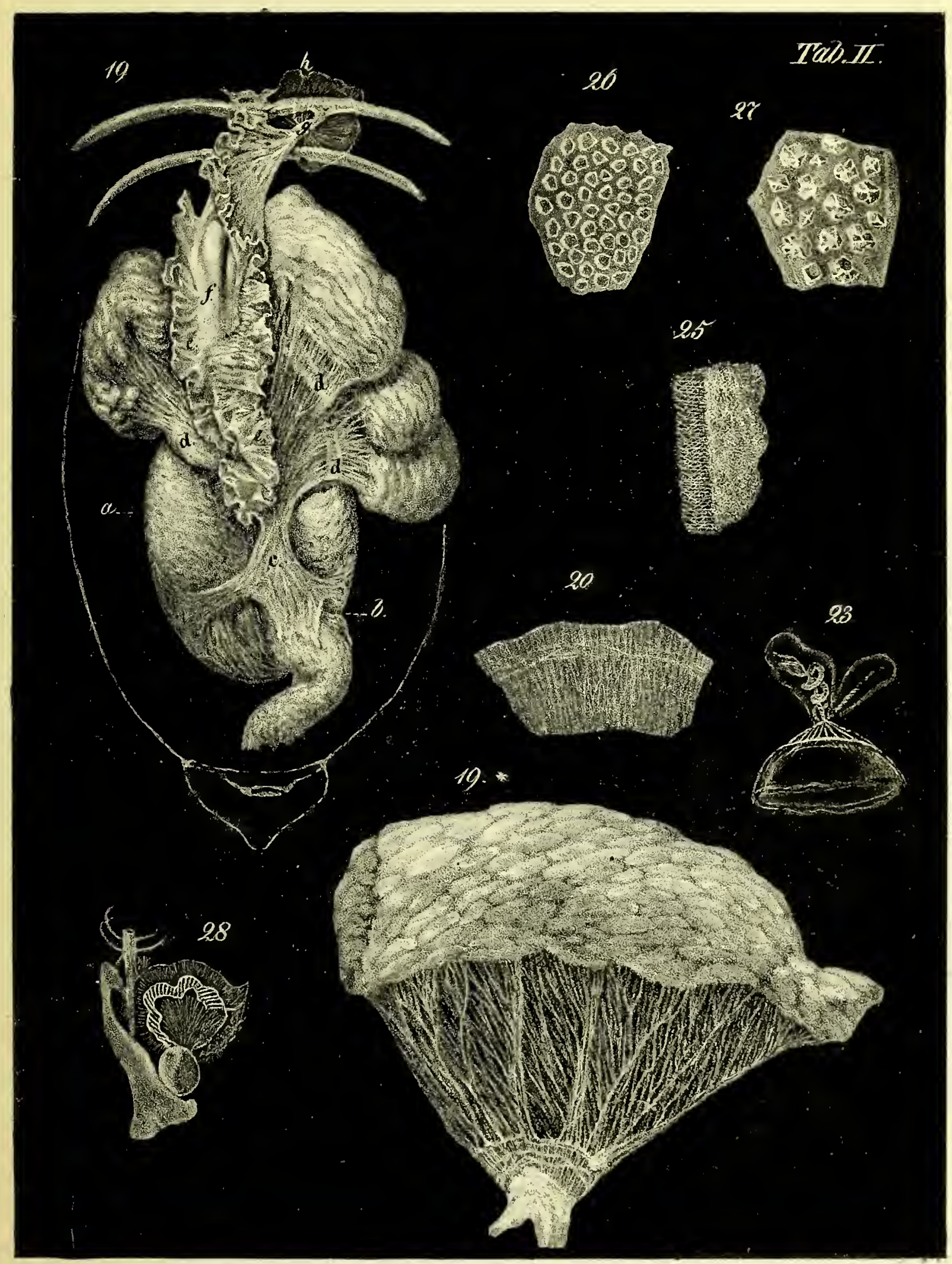

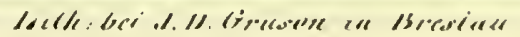




\section{、}


































, 









- 





















































